\title{
Modelling fiber drawing: capillary manufacture
}

\author{
A.D. Fitt ${ }^{*}$, C.P. Please ${ }^{*}$, K. Furusawa ${ }^{\dagger}$ and T.M. Monro ${ }^{\dagger}$ \\ Faculty of Mathematical Studies, ${ }^{\dagger}$ Optoelectronics Research Centre, University of Southampton, UK
}

\begin{abstract}
An asymptotic fluid mechanics model has been posed for the drawing of capillaries. The theory not only gives good agreement with experiment, but provides insight that ispractically relevant to holey fiber manufacture.
\end{abstract}

In the past few years an important new class of optical fiber has emerged: the holey or microstructured fiber. These fibers possess unusual and tailorable optical properties, for example, they can be single mode at all wavelengths [1], have mode areas ranging over three orders of magnitude [2] and display anomalous dispersion at visible wavelengths. Microstructured fibers are produced by drawing a macroscopic preform into fiber using a drawing tower, and the geometry of the final fiber can be modified by controlling the drawing process. For example, at high temperatures, the holes reduce in size because of surface tension effects. In this way a range of fibers with dramatically different optical properties can be produced from one preform.

Although extensive theoretical and numerical work has been performed on the drawing of conventional solid fibers (see for example [3] and [4]) no detailed theory has yet been proposed for fibers that have a crosssection containing holes. Even the simplest case of a single annular capillary has not yet been modeled. Since the optical properties of a microstructured fiber depend critically upon the hole arrangement, it is important to be able to predict how the fabrication parameters influence the final cross-section. In order to accomplish this, we have developed a theoretical framework to study the problem of capillary drawing which is also of practical importance since it is a crucial step in the fabrication of more complex microstructured fibers. To develop a model that is capable of including effects such as surface tension, varying viscosity and internal hole overpressure, we begin from the Navier-Stokes equations. For details of this model, please see Ref. [6]. Asymptotic analysis has been used to simplify these equations for a number of regimes of physical interest, and we present here some of the useful insights provided by this approach.

For the steady-state problem, if gravity and inertia are ignored (they do not contribute at leading order for the parameters considered in our experimental study), it is possible to derive an exact solution to this problem that incorporates viscosity and surface tension effects in the limit of small surface tension:

$$
\begin{aligned}
& u_{0}(x)=U_{f} e^{\beta \bar{x}}+2 \alpha e^{\beta \bar{x}}\left[e^{-\beta \bar{x} / 2}-1+\bar{x}\left(1-e^{-\beta / 2}\right)\right], \\
& \frac{h_{1}(x)}{h_{10}}=e^{-\beta \bar{x} / 2}+\alpha e^{-\beta \bar{x}}\left[\left(\frac{3 h_{20}}{h_{10}}-1\right)\left(1-e^{\beta \bar{x} / 2}\right)+\bar{x} e^{\beta \bar{x} / 2}\left(e^{-\beta / 2}-1\right)\right], \\
& \frac{h_{2}(x)}{h_{20}}=e^{-\beta \bar{x} / 2}+\alpha e^{-\beta \bar{x}}\left[\left(\frac{3 h_{10}}{h_{20}}-1\right)\left(1-e^{\beta \bar{x} / 2}\right)+\bar{x} e^{\beta \bar{x} / 2}\left(e^{-\beta / 2}-1\right)\right],
\end{aligned}
$$

where $\bar{x}=x / L, \dot{\beta}=\ln \left(U_{d} / U_{f}\right)$ and $\alpha=\gamma L /\left(3 \mu \beta U_{f}\left(h_{20}-h_{10}\right)\right)$. Here $x$ measures the distance along the capillary, $U_{f}$ and $U_{d}$ are the feed and draw speeds, dynamic viscosity and surface tension are $\mu$ and $\gamma$ respectively and the glass velocity in the $\mathrm{x}$-direction is $u(x)$. The inner and outer radii of the capillary are denoted by $h_{1}(x)$ and $h_{2}(x)$ respectively, and the boundary conditions are $h_{1}(0)=h_{10}, h_{2}(0)=h_{20}, u_{0}(0)=$ $U_{f}, u_{0}(L)=U_{d}$ where $L$ denotes the length over which the preform is heated in the furnace. The perturbation solution (1)-(3) has the advantage that the phenomenological behaviour of the solution is readily apparent.

To check the validity of the model we performed drawing experiments using a preform tube with an outer diameter (OD) of $28 \mathrm{~mm}$ and an inner diameter (ID) of $24 \mathrm{~mm}$. The glass used was Suprasil F300, a grade of silica commonly used for low-loss fibers. The preform was fed at a constant speed into the graphite furnace with a $3 \mathrm{~cm}$ "hot zone" $(L)$ and the top of the tube was left open. A diameter gauge located approximately $1 \mathrm{~m}$ below the furnace was used to monitor the final diameter of the capillary. For the study 24 experimental runs were carried out: the feed speed $U_{f}$ was varied between 2 and $8 \mathrm{~mm} / \mathrm{min}$, the draw speed $U_{d}$ was varied 
from 0.6 to $1.2 \mathrm{~m} / \mathrm{min}$ and furnace temperatures of 1900,1950 and $2000^{\circ} \mathrm{C}$ were used. Once any particular combination of drawing conditions was set, the process was allowed to stabilise prior to measurement. The theoretical predictions from (3) and (2) are indicated by solid lines in Fig. 1. The comparison between the theory and the experimental results is striking. The inset in Fig.1(b) shows that the experiments were not geometry-preserving and some collapse occurred from the original diameter ratio of $24 / 28 \approx 0.857$.
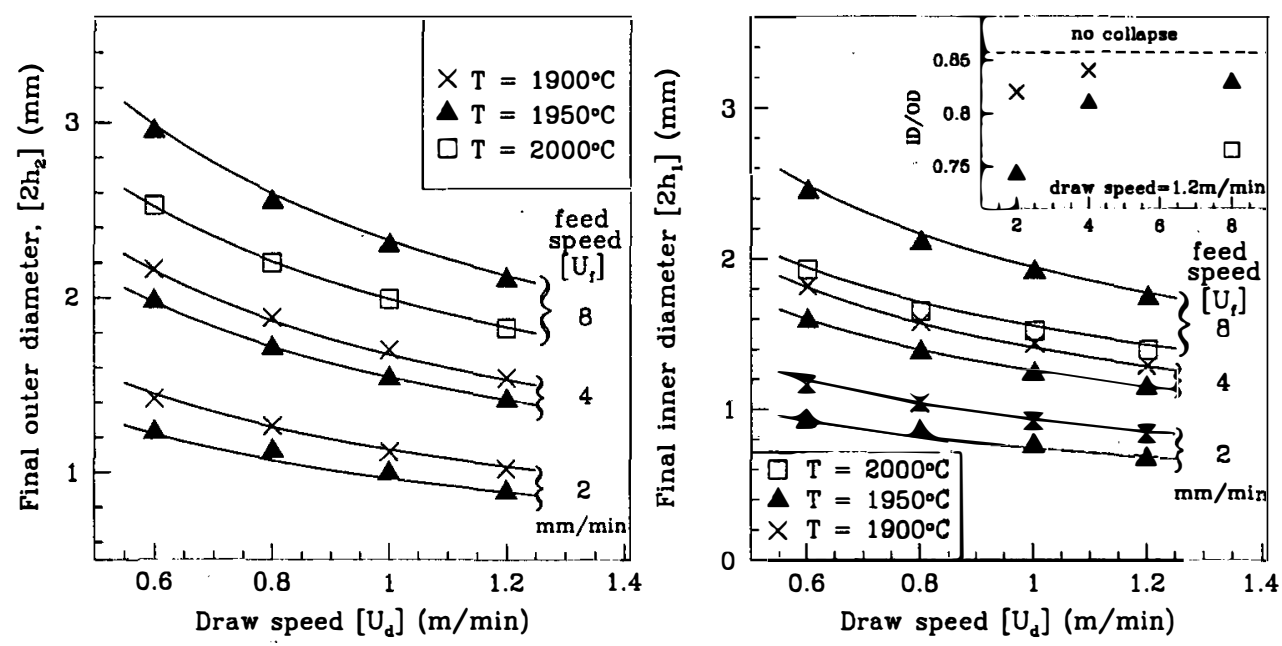

Fig. 1. Experimental results (symbols) and theoretical predictions using Eq. (3) (solid lines) for drawn OD (a) and Eq. ( 2 for drawn ID (b) as functions of draw speed for a range of furnace temperatures and feed speeds. Inset to (b): Drawn diameter ratio for draw speed of $1.2 \mathrm{~m} / \mathrm{min}$ for various furnace temperatures.

One way of controlling the degree of hole closure is to introduce a pressure difference $\left(p_{0}\right)$ between air inside and outside the capillary. If inertia, gravity and surface tension are ignored, we again find an exact solution:

$$
\frac{h_{1}^{2}}{h_{2}^{2}}=a^{2} \exp \left[\frac{p_{0} L\left(1-e^{-\beta \bar{x}}\right)}{U_{f} \mu \beta}\right]
$$

and thus the geometry is no longer preserved. In some circumstances the capillary can explode, and Eq. (4) is useful for estimating the range of pressures that can be used to control the fabrication process: pressure is a useful control when: $p_{0}<2 U_{f} \mu \beta \log a /\left(L\left(e^{-\beta}-1\right)\right)$. For the case of small holes, simple conditions have also been derived for the conditions required to balance viscosity, surface tension and pressure effects and thus preserve the geometry during drawing. These results have been validated against experimental data.

This asymptotic fluid dynamics approach has proved to be a useful model for the drawing of capillaries, and yields a range of practical guidances for the design and control of capillary manufacture. This can be regarded as a first step in the quantification of the process of drawing arbitrary shaped holey fibers.

1. T. A. Birks, J. C. Knight, and P. St. J. Russell, "Endlessly single-mode photonic crystal fiber," Opt. Lett. 22, 961-963 (1997).

2. T. M. Monro, D. J. Richardson, N. G. R. Broderick, and P. J. Bennett, "Holey optical fibers: An efficient modal model," J. Lightwave Technol. 17, 1093-1102 (1999).

3. M. A. Matovich, and J. R. A Pearson, "Spinning a molten threadline - Steady-state isothermal viscous flows," Ind. Eng. Chem. Fund. 8, 512-520 (1969).

4. H. Papamichael, and I. N. Miaoulis, "Thermal-behavior of optical fibers during the cooling stage of the drawing process," J. Mater. Res. 6, 159-167 (1991).

5. S. H.-K. Lee, and Y. Jaluria, "Simulation of the transport processes in the neck-down region of a furnace drawn optical fiber," Int. J. Heat Mass Transfer 40, 843-856 (1997).

6. A. D. Fitt, K. Furusawa, T. M. Monro, and C. P. Please, "Modeling the Fabrication of Hollow Fibers", to appear in J. Lightwave Technol. 19, 2001. 